\title{
Notes on lattice observables for parton distributions: nongauge theories
}

\author{
Luigi Del Debbio, ${ }^{a}$ Tommaso Giani $^{a}$ and Christopher J. Monahan ${ }^{b, c}$ \\ ${ }^{a}$ The Higgs Centre for Theoretical Physics, The University of Edinburgh, \\ Peter Guthrie Tait Road, Edinburgh EH9 3FD, U.K. \\ ${ }^{b}$ Physics Department, William \& Mary, \\ 300 Ukrop Way, Williamsburg, VA 23187, U.S.A. \\ ${ }^{c}$ Thomas Jefferson National Accelerator Facility, \\ 12000 Jefferson Ave, Newport News, VA 23606, U.S.A. \\ E-mail: luigi.del.debbio@ed.ac.uk, tommaso.giani@ed.ac.uk, \\ cjmonahan@wm. edu
}

ABSTRACT: We review recent theoretical developments concerning the definition and the renormalization of equal-time correlators that can be computed on the lattice and related to Parton Distribution Functions (PDFs) through a factorization formula. We show how these objects can be studied and analyzed within the framework of a nongauge theory, gaining insight through a one-loop computation. We use scalar field theory as a playground to revise, analyze and present the main features of these ideas, to explore their potential, and to understand their limitations for extracting PDFs. We then propose a framework that would allow to include the available lattice QCD data in a global analysis to extract PDFs.

KEYWORDS: Lattice field theory simulation, QCD Phenomenology

ARXiv EPRINT: 2007.02131 


\section{Contents}

1 Introduction 1

2 Light-cone separation $\quad 3$

$\begin{array}{llr}3 & \text { Spatial separation } & 7\end{array}$

4 Factorization theorem $\quad 8$

4.1 Factorization theorem in position space: small- $z_{3}^{2}$ limit 9

4.2 Factorization theorem in momentum space: large $P_{3}$ limit $\quad 10$

$\begin{array}{lll}5 & \text { Smeared distributions } & 12\end{array}$

6 Conclusions $\quad 15$

$\begin{array}{ll}\text { A Momentum space factorization } & 16\end{array}$

$\begin{array}{ll}\text { B Equivalence between pseudo- and quasi-PDF approaches } & 18\end{array}$

$\begin{array}{ll}\text { C Quasi-PDFs and their moments } & 19\end{array}$

\section{Introduction}

In recent years, there has been a significant effort within the lattice community to compute specific equal-time correlators that can be directly related to Parton Distribution Functions (PDFs). PDFs describe the longitudinal structure of nucleons in terms of their partonic constituents. They are inherently non-perturbative quantities, which can be extracted from data using so-called factorization theorems. Given the central role of PDFs in the analysis of experimental data at hadronic colliders, it would be highly beneficial to be able to use lattice QCD to determine these crucial ingredients in our current understanding of nucleon structure. Quasi-PDFs ${ }^{1}$ and pseudo-PDFs were introduced in refs. [3, 4], and since then numerous publications have appeared, addressing the main theoretical issues for these approaches. For recent reviews, we refer the reader to refs. [2, 5-10]. This program has often been referred to as the "first principles computation of PDFs", generating different reactions among the lattice and high-energy physics communities: on the one hand it has been welcomed with enthusiasm, triggering several dedicated studies; on the other hand it has been criticized in refs. [11, 12] on the basis that equal-time correlators do not give access to the full non-perturbative PDF. Both reactions are healthy and show the importance of

\footnotetext{
${ }^{1}$ Quasi-PDFs are one example of the more general LaMET formalism $[1,2]$, but here we focus on the collinear $x$-dependent distributions.
} 
the original proposal in [3]. This criticism mentioned above has, in turn, been addressed in refs. $[13,14]$. Given the increasing number of lattice calculations, there is a need to revise and clarify the main conceptual questions: that is, how do we extract information on PDFs from quasi- and pseudo-PDFs, and what is the interplay between quasi- and pseudo-PDFs with experimental data?

In this paper we study these topics in the context of a renormalizable scalar theory. Scalar field theory is a valuable model for understanding the essential theoretical issues in a simple framework, as shown in the pioneering study of PDFs by Collins in ref. [15]. We follow the ideas presented there, which we extend to account for quasi- and pseudoPDFs. Our aim is to investigate, clarify and highlight some subtle points using scalar field theory as a simple playground, and to assess how the lattice QCD results that are currently available can be used to extract PDFs.

We will consider a massive scalar field theory, in $d=6$ dimensions, with a $\phi^{3}$ interaction term, whose bare Lagrangian $\mathcal{L}$ is given by

$$
\mathcal{L}=\frac{1}{2}(\partial \phi)^{2}-\frac{m^{2}}{2} \phi^{2}-\frac{g}{3 !} \phi^{3} .
$$

Working within this model allows us to analyze the conceptual framework for quasi- and pseudo-PDFs in a clean and straightforward way, avoiding complications associated with QCD that are unnecessary for understanding the basics of these approaches. We focus in particular on the matrix element of a field bilinear between "nucleon" states:

$$
\mathcal{M}=\langle P|\phi(z) \phi(0)| P\rangle,
$$

when the separation $z$ between the fields is either light-cone like, $z^{2}=0$, or purely spatial, $z^{2}=-z_{3}^{2}$. In the first case, we obtain the matrix element that underlies the formal definition of collinear PDFs $[15,16]$, which are obtained as the Fourier transform along a light-cone direction of the matrix element in eq. (1.2): ${ }^{2}$

$$
f(x)=x P^{+} \int \frac{d z^{-}}{2 \pi} e^{-i x P^{+} z^{-}}\langle P|\phi(z) \phi(0)| P\rangle,
$$

where $P^{+}$and $z^{-}$are the usual light-cone coordinates of the four-vectors $P$ and $z$ respectively. In the second case we obtain an equal-time correlator that can be computed on the lattice. We address the problem of the renormalization of these quantities and study the relation between them at one loop in perturbation theory, both in position and momentum space. As we shall see, the main features of the computation are the same as in QCD. This allows us to understand easily the main concepts, relations and limitations of the quasi- and pseudo-PDF approaches. With a clear picture of the theoretical background and of what is currently available in the literature, we then propose a general framework to extract collinear PDFs from the available lattice data, based on the optimization of a parametric form of the PDFs. The implementation of such approach has been started in ref. [17] within the NNPDF environment, using the same strategy that is commonly used to extract PDFs from data for experimental observables.

\footnotetext{
${ }^{2}$ The field bilinear needs to undergo a proper renormalization, which we explore in detail in this paper.
} 
We address, in turn, a number of questions that have been raised in the context of QCD, and analyze the lessons that we can draw from the scalar model.

First we discuss issues that are related to the analysis of ultraviolet (UV) divergences of the bilinear operator and their subtraction through the renormalization process. In particular in section 2 we perform the computation of $\mathcal{M}$ in the case of a light-cone separation, recovering the results of ref. [15] through a position space calculation. In section 3 we perform the same exercise outside the light-cone, choosing a purely spatial separation between fields, and we discuss the main differences with respect to the light-cone case.

In both cases, we define quantities that are free of divergences when the regulator is removed, and then focus on the relation between light-cone and equal-time correlators. In section 4 we work out this relation explicitly at one loop in perturbation theory, and analyze the limits leading to a factorization theorem, in both position and momentum space, and in section 5 we extend the discussion to include smeared equal-time correlators.

In section 6 we summarize, discuss how these ideas can be used in a fitting framework to extract PDFs, and draw our conclusions. The work is supplemented with a number of appendices containing the technical details of the computations and addressing the objections raised in refs. [11, 12].

\section{Light-cone separation}

As stressed in ref. [4], the matrix element defined in eq. (1.2) is a function of the Lorentz invariants $z^{2}$ and $\nu=P \cdot z$, the "Ioffe time", so that we can write $\mathcal{M}=\mathcal{M}\left(\nu, z^{2}\right)$. In this section we focus on the perturbative renormalization of $\mathcal{M}\left(\nu, z^{2}\right)$ at the one-loop level, in the light-cone separation case, $z^{2}=0$. We work in perturbation theory, denoting the bare field of our theory as $\phi$, and we consider partonic matrix elements

$$
\widehat{\mathcal{M}}\left(\nu, z^{2}\right)=\langle p|\phi(z) \phi(0)| p\rangle
$$

between on-shell quark states with four-momentum $p$, with $p^{2}=m_{\text {pole }}^{2}$. Throughout this calculation, we denote partonic quantities with a "hat", while the lower-case $p$ refers to the momentum of the parton. In what follows the Lorentz invariant $\nu$ is defined as $\nu=p \cdot z$. Restricting ourselves to the case for which $z_{0} \geqslant 0$, we have

$$
\begin{aligned}
& \widehat{\mathcal{M}}\left(\nu, z^{2}\right)=\langle p|T[\phi(z) \phi(0)]| p\rangle \\
& \quad=\lim _{p^{2} \rightarrow m_{\text {pole }}^{2}}\left(p^{2}-m_{\text {pole }}^{2}+i \epsilon\right)^{2} \int d z_{1} d z_{2} e^{-i p \cdot z_{1}} e^{i p \cdot z_{2}}\left\langle 0\left|T\left[\phi(z) \phi(0) \phi\left(z_{1}\right) \phi\left(z_{2}\right)\right]\right| 0\right\rangle
\end{aligned}
$$

where $m_{\text {pole }}^{2}$ is defined by the location of the pole in the scalar propagator, and can be computed at each order in perturbation theory. At tree level we have $m_{\text {pole }}^{2}=m^{2}$, while in general $m_{\text {pole }}^{2}-m^{2}=\mathcal{O}\left(g^{2}\right)$.

When computing the 4-point function entering eq. (2.2), we will not consider diagrams like those in figure 1. Following ref. [15], we are only interested in the contribution proportional to $\exp (-i p \cdot z)$, and therefore discard topologies like the one in diagram (a). Diagram (b) is removed by considering the connected contribution only. 


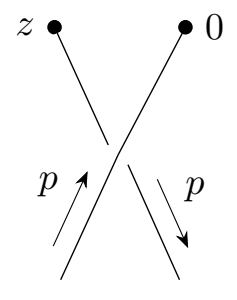

(a)

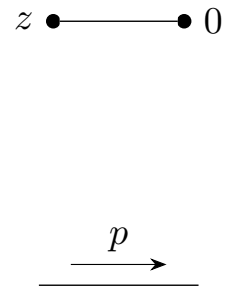

(b)

Figure 1. Contractions that are not considered in the present discussion. Diagram (a) is excluded when considering contributions proportional to $\exp (-i p \cdot z)$, while diagram (b) cancels when looking at the connected correlator.

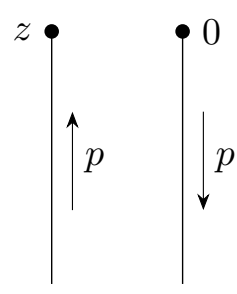

(a)

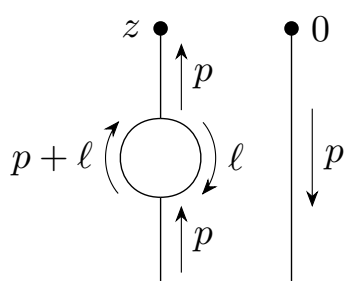

(b)

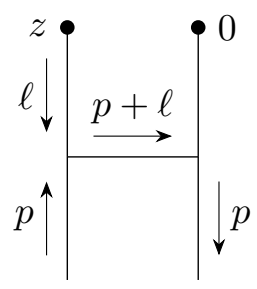

(c)

Figure 2. Feynman diagrams up to one loop for $\left\langle 0\left|T\left[\phi(z) \phi(0) \phi\left(z_{1}\right) \phi\left(z_{2}\right)\right]\right| 0\right\rangle$.

Therefore the only Feynman diagrams contributing to eq. (2.2) up to one-loop order are those shown in figure 2. Denoting the propagator in position space as

$$
\langle 0|T[\phi(x) \phi(y)]| 0\rangle=\overparen{\phi}_{x} \phi_{y},
$$

the Wick contraction that contributes to the tree level diagram (a) of figure 2 is given by

$$
\varlimsup_{z} \phi_{z_{1}} \overbrace{z_{2}} \phi_{0}=\int_{l_{1}} \frac{i e^{-i l_{1} \cdot\left(z-z_{1}\right)}}{l_{1}^{2}-m^{2}+i \epsilon} \int_{l_{2}} \frac{i e^{-i l_{2} \cdot z_{2}}}{l_{2}^{2}-m^{2}+i \epsilon},
$$

where we use the notation

$$
\int_{k}=\int \frac{\mathrm{d}^{d} k}{(2 \pi)^{d}}
$$

Plugging eq. (2.4) in eq. (2.2) we obtain the tree level expression for $\widehat{\mathcal{M}}\left(\nu, z^{2}\right)$

$$
\widehat{\mathcal{M}}^{(0)}\left(\nu, z^{2}\right)=-e^{-i \nu} \equiv \widehat{\mathcal{M}}^{(0)}(\nu, 0) .
$$

Note that the tree level result does not depend on the invariant separation $z^{2}$ and therefore we can set $z^{2}=0$ in the second equality above.

At one-loop order the self-energy diagram (b) yields the mass and wave function renormalization. Its contribution to eq. (2.2) is

$$
\widehat{\mathcal{M}}_{\text {self }}\left(\nu, z^{2}\right)=R \widehat{\mathcal{M}}^{(0)}(\nu, 0),
$$


where $R$ is the $\mathcal{O}\left(g^{2}\right)$ contribution to the residue of the propagator at the pole mass. In $d=6-2 \epsilon$ dimensions, we have

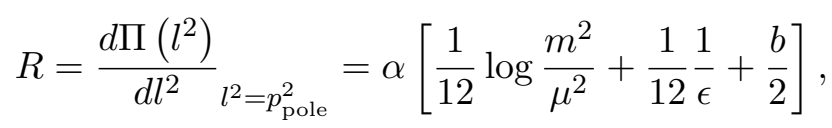

where $b / 2$ is a finite contribution and $\alpha=g^{2} /\left(64 \pi^{3}\right)$. The same $\mathcal{O}(\alpha)$ contribution is obtained from the diagram with the self energy corrections on the second leg, so that the total contribution coming from the tree level plus self-energy corrections is

$$
\widehat{\mathcal{M}}_{\text {self }}\left(\nu, z^{2}\right)=\left[1+\alpha\left(\frac{1}{6} \log \frac{m^{2}}{\mu^{2}}+\frac{1}{6} \frac{1}{\epsilon}+b\right)\right] \widehat{\mathcal{M}}^{(0)}(\nu, 0)+\mathcal{O}\left(\alpha^{2}\right)
$$

Note the absence of any $z^{2}$ dependence: as far as the first two diagrams of figure 2 are concerned, there are no differences between the light-cone and the pure spatial case. This is to be expected, since the one-loop diagrams (b) simply implement the mass and wave function renormalization.

We can now move to the computation of the remaining $\mathcal{O}(\alpha)$ term, i.e. diagram (c). This contraction is given by

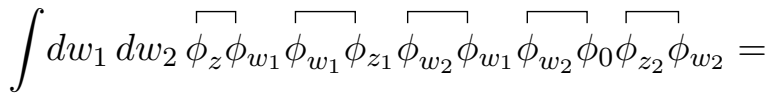

$$
\begin{aligned}
& =(-i g)^{2} \int d w_{1} d w_{2} \int_{l_{1}} \frac{i e^{-i l_{1} \cdot\left(z-w_{1}\right)}}{l_{1}^{2}-m^{2}+i \epsilon} \int_{l_{2}} \frac{i e^{-i l_{2} \cdot\left(w_{1}-z_{1}\right)}}{l_{2}^{2}-m^{2}+i \epsilon} \int_{l_{3}} \frac{i e^{-i l_{3} \cdot\left(w_{2}-w_{1}\right)}}{l_{3}^{2}-m^{2}+i \epsilon} \times \\
& \times \int_{l_{4}} \frac{i e^{-i l_{4} \cdot w_{2}}}{l_{4}^{2}-m^{2}+i \epsilon} \int_{l_{5}} \frac{i e^{-i l_{5} \cdot\left(z_{2}-w_{2}\right)}}{l_{5}^{2}-m^{2}+i \epsilon} \text {. }
\end{aligned}
$$

Plugging this into eq. (2.2), we have

$$
\begin{aligned}
\widehat{\mathcal{M}}^{(1)}\left(\nu, z^{2}\right) & =-i g^{2} \int_{k} \frac{e^{-i k \cdot z}}{\left(k^{2}-m^{2}+i \epsilon\right)^{2}} \frac{1}{(p-k)^{2}-m^{2}+i \epsilon} \\
& =g^{2} \int_{0}^{1} d \xi(1-\xi) K\left(z^{2}, M^{2}\right) \widehat{\mathcal{M}}^{(0)}(\xi \nu, 0),
\end{aligned}
$$

where we have introduced a Feynman parameter $\xi$ and defined

$$
K\left(z^{2}, M^{2}\right)=2 i \int_{q} \frac{e^{-i q \cdot z}}{\left(q^{2}-M^{2}+i \epsilon\right)^{3}},
$$

with

$$
\begin{aligned}
q & =k-\xi p \\
M^{2} & =m^{2}\left(1-\xi+\xi^{2}\right) .
\end{aligned}
$$

The integral $K\left(z^{2}, M^{2}\right)$ can be computed by performing a Wick rotation $z_{E}^{\mu}=\left(i z^{0}, \vec{z}\right)$ and using

$$
\frac{1}{\left(q_{E}^{2}+m^{2}\right)^{\alpha}}=\frac{1}{\Gamma(\alpha)} \int_{0}^{\infty} d T T^{\alpha-1} e^{-T\left(q_{E}^{2}+m^{2}\right)} .
$$


We obtain

$$
\begin{aligned}
K\left(z^{2}, M^{2}\right) & =2 \int \frac{d^{d} q_{E}}{(2 \pi)^{d}} \frac{e^{i q_{E} z_{E}}}{\left(q_{E}^{2}+M^{2}\right)^{3}}=\int_{0}^{\infty} d T T^{2} e^{-T M^{2}} \int \frac{d^{d} q_{E}}{(2 \pi)^{d}} e^{i q_{E} z_{E}-T q_{E}^{2}} \\
& =\frac{1}{(4 \pi)^{\frac{d}{2}}} \int_{0}^{\infty} \frac{d T}{T} T^{3-\frac{d}{2}} e^{-T M^{2}} e^{-\frac{z_{E}^{2}}{4 T}}
\end{aligned}
$$

where in the last line we have performed the Gaussian integral over $d^{d} q_{E}$.

Since we are considering the case of a light-cone separation $z_{E}^{2}=-z^{2}=0, K\left(0, M^{2}\right)$ in $d=6$ dimensions is logarithmically divergent. The divergence arises from the lower end of the integral over $T$, as the exponential suppression factor in the integrand vanishes on the light-cone. We apply dimensional regularization, taking $d=6-2 \epsilon$ and introducing the $\overline{\mathrm{MS}}$ scale $\mu$ through the rescaling of the coupling $g^{2} \rightarrow g^{2} e^{\gamma_{E}} \mu^{2} /(4 \pi)$. We find

$$
K\left(0, M^{2} ; \mu^{2}\right)=\int_{0}^{\infty} \frac{d T}{T}\left(T \mu^{2} e^{\gamma_{E}}\right)^{\epsilon} e^{-T M^{2}}=\Gamma(\epsilon)\left(\frac{\mu^{2} e^{\gamma_{E}}}{M^{2}}\right)^{\epsilon}=\frac{1}{\epsilon}+\log \frac{\mu^{2}}{M^{2}},
$$

where the pole in $1 / \epsilon$ reflects the original logarithmic divergence in dimensional regularization. Putting everything together, we obtain the full one-loop expression of the bare position space matrix element in dimensional regularization

$$
\begin{aligned}
\widehat{\mathcal{M}}(\nu, 0)= & {\left[1+\alpha\left(\frac{1}{6} \log \frac{m^{2}}{\mu^{2}}+\frac{1}{6} \frac{1}{\epsilon}+b\right)\right] \widehat{\mathcal{M}}^{(0)}(\nu, 0) } \\
& +\alpha \int_{0}^{1} d \xi(1-\xi)\left(\frac{1}{\epsilon}+\log \frac{\mu^{2}}{m^{2}\left(1-\xi+\xi^{2}\right)}\right) \widehat{\mathcal{M}}^{(0)}(\xi \nu, 0) .
\end{aligned}
$$

The structure of the divergences in eq. (2.18) shows that this quantity can be renormalized by convolution with a renormalization kernel $\mathcal{K}$. Denoting the renormalized matrix element as $\widehat{\mathcal{M}}_{R}\left(\nu, 0, \mu^{2}\right)$, we have

$$
\widehat{\mathcal{M}}_{R}\left(\nu, 0, \mu^{2}\right)=\int_{0}^{1} d y \mathcal{K}(y) \widehat{\mathcal{M}}(y \nu, 0) .
$$

The specific choice of the finite terms that appear in the kernel $\mathcal{K}(y)$, together with subtraction of the $1 / \epsilon$ poles, defines the renormalization scheme. For example, in the $\overline{\mathrm{MS}}$ scheme, the renormalization kernel is

$$
\mathcal{K}(y)=\delta(1-y)-\alpha\left[\frac{1}{6 \epsilon} \delta(1-y)+\frac{1}{\epsilon}(1-y)\right],
$$

and the corresponding renormalized quantity is

$$
\begin{aligned}
\widehat{\mathcal{M}}_{R}\left(\nu, 0, \mu^{2}\right)= & {\left[1+\alpha\left(\frac{1}{6} \log \frac{m^{2}}{\mu^{2}}+b\right)\right] \widehat{\mathcal{M}}^{(0)}(\nu, 0) } \\
& +\alpha \int_{0}^{1} d \xi(1-\xi) \log \frac{\mu^{2}}{m^{2}\left(1-\xi+\xi^{2}\right)} \widehat{\mathcal{M}}^{(0)}(\xi \nu, 0) .
\end{aligned}
$$

We conclude this derivation with a comment on the form of the renormalization kernel $\mathcal{K}$ given in eq. (2.20): the contribution proportional to a delta function is a multiplicative 
renormalization term, implementing the subtraction of the singularities generated by diagram (b) of figure 2, which is basically the wave function renormalization. The second contribution, $-\frac{\alpha}{\epsilon}(1-y)$, implements the renormalization of the one-loop diagram (c) of figure 2, and because this contribution is not proportional to a delta function, the renormalization of this term is not multiplicative, but requires a convolution.

Taking the log derivative of eq. (2.21) we obtain

$$
\mu^{2} \frac{d}{d \mu^{2}} \widehat{\mathcal{M}}_{R}\left(\nu, 0, \mu^{2}\right)=\alpha \int_{0}^{1} d \xi P(\xi) \widehat{\mathcal{M}}_{R}\left(\xi \nu, 0, \mu^{2}\right)+\mathcal{O}\left(\alpha^{2}\right)
$$

where the $\mathcal{O}(\alpha)$ Altarelli-Parisi splitting kernel is given by

$$
P(\xi)=(1-\xi)-\frac{1}{6} \delta(1-\xi)=(1-\xi)_{+}+\frac{1}{3} \delta(1-\xi),
$$

with the action of the plus distribution over a generic test function $g(\xi)$ defined as

$$
\int_{0}^{1} d x(1-\xi)_{+} g(\xi)=\int_{0}^{1} d x(1-\xi)[g(\xi)-g(1)] .
$$

The renormalized collinear PDF is defined from the renormalized matrix element,

$$
\widehat{\mathcal{M}}_{R}\left(\nu, 0, \mu^{2}\right)=\int_{0}^{1} d x e^{i x \nu} \widehat{f}\left(x, \mu^{2}\right)
$$

and therefore, from eq. (2.22),

$$
\mu^{2} \frac{d}{d \mu^{2}} \widehat{f}\left(x, \mu^{2}\right)=\alpha \int_{x}^{1} \frac{d \xi}{\xi} P(\xi) \widehat{f}\left(\frac{x}{\xi}, \mu^{2}\right),
$$

which yields the standard DGLAP evolution equations, which were already obtained in ref. [15] for the scalar theory. The solution of eq. (2.26) in perturbation theory is given by an evolution kernel $\Gamma\left(x, \mu, \mu_{0}, \alpha\right)$, which allows the PDF at a generic scale $\mu$ to be computed in terms of the PDF at the scale $\mu_{0}$ as

$$
\widehat{f}\left(x, \mu^{2} ; \theta\right)=\int_{x}^{1} \frac{d \xi}{\xi} \Gamma\left(\frac{x}{\xi}, \mu, \mu_{0}, \alpha_{s}\right) \widehat{f}\left(\xi, \mu_{0}^{2} ; \theta\right) .
$$

\section{Spatial separation}

We now consider the case in which the separation between the fields is purely spatial $z_{E}^{2}=z_{3}^{2}$. As seen in the previous section, the $z^{2}$ dependence enters only through diagram (c) of figure 2. Considering this contribution, the kernel $K\left(z^{2}, M^{2}\right)$ defined in eq. (2.16) is no longer divergent for $z_{3} \neq 0$, as the term $\exp \left[-z_{E}^{2} /(4 T)\right]$ regulates the small- $T$ behaviour. The integral can evaluated directly in $d=6$ dimensions, yielding

$$
K\left(-z_{3}^{2}, M^{2}\right)=\frac{1}{64 \pi^{3}} \int_{0}^{\infty} \frac{d T}{T} e^{-T} e^{-\frac{\left(M z_{3}\right)^{2}}{4 T}}=\frac{1}{64 \pi^{3}} 2 K_{0}\left(M z_{3}\right)
$$


where $K_{0}$ is the modified Bessel function. Plugging eq. (3.1) into eq. (2.11) we obtain the contribution from diagram (c) in the case of purely spatial separation:

$$
\widehat{\mathcal{M}}^{(1)}\left(\nu,-z_{3}^{2}\right)=\alpha \int_{0}^{1} d \xi(1-\xi) 2 K_{0}\left(M z_{3}\right) \widehat{\mathcal{M}}^{(0)}(\xi \nu, 0) .
$$

Note that, as long as $z_{3} \neq 0$, this contribution does not contain any UV divergences. For $M z_{3} \rightarrow 0$ the Bessel function diverges logarithmically, and we recover the UV divergence of the light-cone case.

The full one-loop bare matrix element is then given by

$$
\begin{aligned}
\widehat{\mathcal{M}}\left(\nu,-z_{3}^{2}\right)= & {\left[1+\alpha\left(\frac{1}{6} \log \frac{m^{2}}{\mu^{2}}+\frac{1}{6} \frac{1}{\epsilon}+b\right)\right] \widehat{\mathcal{M}}^{(0)}(\nu, 0) } \\
& +\alpha \int_{0}^{1} d \xi(1-\xi) 2 K_{0}\left(M z_{3}\right) \widehat{\mathcal{M}}^{(0)}(\xi \nu, 0) .
\end{aligned}
$$

As before, this quantity can be renormalized by convolution,

$$
\widehat{\mathcal{M}}_{R}\left(\nu,-z_{3}^{2} ; \mu^{2}\right)=\int_{0}^{1} d \xi \tilde{\mathcal{K}}(\xi) \widehat{\mathcal{M}}\left(\xi \nu,-z_{3}^{2}\right) .
$$

However, since the only UV pole comes from the self-energy contributions, the kernel $\tilde{\mathcal{K}}(\xi)$ is proportional to a delta function. For example, in the $\overline{\mathrm{MS}}$ scheme we can take

$$
\tilde{\mathcal{K}}(\xi)=\delta(1-\xi)\left[1-\alpha \frac{1}{6 \epsilon}\right] .
$$

In other words, in the case of purely spatial separation the renormalization of the matrix element is purely multiplicative [18]. The additional UV divergence we had to remove in the light-cone case is substituted here by a finite contribution $K_{0}\left(M z_{3}\right)$. The corresponding renormalized quantity is

$$
\begin{aligned}
\widehat{\mathcal{M}}_{R}\left(\nu,-z_{3}^{2} ; \mu^{2}\right)= & {\left[1+\alpha\left(\frac{1}{6} \log \frac{m^{2}}{\mu^{2}}+b\right)\right] \widehat{\mathcal{M}}^{(0)}(\nu, 0) } \\
& +\alpha \int_{0}^{1} d \xi(1-\xi) 2 K_{0}\left(M z_{3}\right) \widehat{\mathcal{M}}^{(0)}(\xi \nu, 0) .
\end{aligned}
$$

Note also that both eqs. (2.21) and (3.6) contain an infrared (IR) divergence regularized by the mass $m$ : in the former the mass is manifest in the log, while in the latter the mass appears in the Bessel function, which diverges logarithmically for $m \rightarrow 0$.

\section{Factorization theorem}

Having defined the renormalized correlators in the previous sections, let us investigate the one-loop relation between the light-cone and the equal-time correlators. Combining eqs. (2.21) and (3.6) we write

$$
\begin{aligned}
\widehat{\mathcal{M}}_{R}\left(\nu,-z_{3}^{2} ; \mu^{2}\right)= & \widehat{\mathcal{M}}_{R}\left(\nu, 0, \mu^{2}\right) \\
& +\alpha \int_{0}^{1} d \xi(1-\xi)\left(2 K_{0}\left(M z_{3}\right)-\log \frac{\mu^{2}}{M^{2}}\right) \widehat{\mathcal{M}}_{R}\left(\xi \nu, 0, \mu^{2}\right),
\end{aligned}
$$


and using eq. (2.25) we find

$$
\widehat{\mathcal{M}}_{R}\left(\nu,-z_{3}^{2} ; \mu^{2}\right)=\int_{0}^{1} d x \tilde{C}\left(x \nu, m z_{3}, \frac{\mu^{2}}{m^{2}}\right) \widehat{f}\left(x, \mu^{2}\right),
$$

with

$$
\tilde{C}\left(x \nu, m z_{3}, \frac{\mu^{2}}{m^{2}}\right)=e^{i x \nu}-\alpha \int_{0}^{1} d \xi(1-\xi)\left(2 K_{0}\left(M z_{3}\right)-\log \frac{\mu^{2}}{M^{2}}\right) e^{i \xi x \nu} .
$$

This expression shows the connection between the collinear PDFs and an equal-time correlator, through a convolution with a perturbative kernel. In general, the latter contains a logarithmic dependence on $\mathrm{m}^{2}$, namely the kernel contains IR singularities. However, as we will see, these singularities cancel exactly when taking a specific limit, leaving an expression free from IR poles, which therefore has the form of a proper factorization theorem. Before discussing this in detail, we recall that, although eq. (4.2) has been worked out in perturbation theory, considering matrix elements between on-shell quark states, the renormalization of the bilocal operators discussed so far does not depend on our choice of specific external states. It follows that eq. (4.2) holds also for external proton states. From now on we will refer to full proton matrix elements rather than partonic ones, removing the symbol ' ', used so far to denote partonic quantities.

\subsection{Factorization theorem in position space: small- $z_{3}^{2}$ limit}

The behavior of the coefficient $\tilde{C}$ in the small- $z_{3}^{2}$ limit is obtained by expanding the Bessel function as

$$
2 K_{0}\left(M z_{3}\right)=-\log \left(M^{2} z_{3}^{2}\right)+2 \log \left(2 e^{-\gamma_{E}}\right)+\mathcal{O}\left(M^{2} z_{3}^{2}\right),
$$

so that eq. (4.2) becomes

$$
\mathcal{M}_{R}\left(\nu,-z_{3}^{2} ; \mu^{2}\right)=\int_{0}^{1} d x \tilde{C}\left(x \nu, \mu^{2} z_{3}^{2}\right) f\left(x, \mu^{2}\right),
$$

with

$$
\tilde{C}\left(x \nu, \mu^{2} z_{3}^{2}\right)=e^{i x \nu}-\alpha \int_{0}^{1} d \xi(1-\xi) \log \left(\mu^{2} z_{3}^{2} \frac{e^{2 \gamma_{E}}}{4}\right) e^{i \xi x \nu}+\mathcal{O}\left(m^{2} z_{3}^{2}\right) .
$$

We note that in this limit the logarithmic behaviour of the Bessel function matches that of the light-cone quantity, so that the two matrix elements display the same IR behaviour: as a result the coefficient $\tilde{C}$ is IR safe, and eq. (4.5) represents a proper factorization theorem connecting a lattice computable quantity on the left hand side with a collinear PDF on the right hand side.

We note that this factorization also applies to the so-called reduced distributions $[19$, 20], the quantities usually determined in lattice calculations in the pseudo-PDF approach, first introduced in ref. [4]. They were originally defined as

$$
\mathfrak{M}\left(\nu,-z_{3}^{2}\right)=\frac{\mathcal{M}_{R}\left(\nu,-z_{3}^{2} ; \mu^{2}\right)}{\mathcal{M}_{R}\left(0,-z_{3}^{2} ; \mu^{2}\right)},
$$


although a double ratio was proposed in [21]. Here we restrict our attention to the ratio defined in eq. (4.7). In the context of our model, using the small- $z_{3}^{2}$ limit of eq. (4.1) we have

$$
\begin{aligned}
\mathfrak{M}\left(\nu,-z_{3}^{2}\right) & =\mathcal{M}_{R}\left(\nu, 0, \mu^{2}\right)-\alpha \log \left(\mu^{2} z_{3}^{2} \frac{e^{2 \gamma_{E}}}{4}\right) \int_{0}^{1} d \xi(1-\xi)\left[\mathcal{M}_{R}\left(\xi \nu, 0, \mu^{2}\right)-\mathcal{M}_{R}\left(\nu, 0, \mu^{2}\right)\right] \\
& =\mathcal{M}_{R}\left(\nu, 0, \mu^{2}\right)-\alpha \log \left(\mu^{2} z_{3}^{2} \frac{e^{2 \gamma_{E}}}{4}\right) \int_{0}^{1} d \xi(1-\xi)_{+} \mathcal{M}_{R}\left(\xi \nu, 0, \mu^{2}\right)
\end{aligned}
$$

and therefore

$$
\mathfrak{M}\left(\nu,-z_{3}^{2}\right)=\int_{0}^{1} d x \tilde{C}_{+}\left(x \nu, \mu^{2} z_{3}^{2}\right) f\left(x, \mu^{2}\right)
$$

with

$$
\tilde{C}_{+}\left(x \nu, \mu^{2} z_{3}^{2}\right)=e^{i x \nu}-\alpha \log \left(\mu^{2} z_{3}^{2} \frac{e^{2 \gamma_{E}}}{4}\right) \int_{0}^{1} d \xi(1-\xi)_{+} e^{i \xi x \nu}+\mathcal{O}\left(m^{2} z_{3}^{2}\right) .
$$

Note the absence of any $\mu^{2}$ dependence on the left hand side of eqs. (4.7) and (4.9): the perturbative dependence on the renormalization scale $\mu^{2}$ cancels exactly in the ratio, leaving a quantity that depends only on the scale $z_{3}^{2}$. More precisely, eqs. (4.9), (4.10) show how, in the small- $z_{3}^{2}$ limit, the renormalization scale dependence of $\mathcal{M}_{R}\left(\nu, 0, \mu^{2}\right)$ generated by diagram (c) is replaced by an equal $z_{3}^{2}$ dependence that can be obtained from the former through the substitution

$$
\mu^{2} \rightarrow \frac{4 e^{-2 \gamma_{E}}}{z_{3}^{2}}
$$

In other words, the factorization formula worked out in this section predicts a logarithmic dependence on $z_{3}^{2}$ for the equal-time correlator, which replaces the analogous logarithmic behaviour of the PDFs on the renormalization scale $\mu^{2}$, predicted by the one-loop DGLAP. Such dependence on $z_{3}^{2}$ should be visible in real lattice QCD data when working in the factorization regime, and indeed it was observed in refs. [20, 21].

\subsection{Factorization theorem in momentum space: large $P_{3}$ limit}

A factorization theorem can also be established working in momentum rather than in position space. Taking the Fourier transform of eq. (4.5) with respect to $z_{3}$ and defining

$$
\begin{aligned}
q\left(y, \mu^{2}, P_{3}^{2}\right) & =\frac{P_{3}}{2 \pi} \int_{-\infty}^{\infty} d z_{3} e^{-i y P_{3} z_{3}} \widehat{\mathcal{M}}\left(P_{3} z_{3},-z_{3}^{2}\right), \\
C\left(\eta, \frac{m^{2}}{x^{2} P_{3}^{2}}, \frac{\mu^{2}}{m^{2}}\right) & =\int_{-\infty}^{\infty} \frac{d \theta}{2 \pi} e^{-i \theta \eta} \tilde{C}\left(\theta, \frac{m \theta}{x P_{3}}, \frac{\mu^{2}}{m^{2}}\right)
\end{aligned}
$$

we obtain

$$
q\left(y, \mu^{2}, P_{3}^{2}\right)=\int_{0}^{1} \frac{d x}{x} f\left(x, \mu^{2}\right) C\left(\frac{y}{x}, \frac{m^{2}}{x^{2} P_{3}^{2}}, \frac{\mu^{2}}{m^{2}}\right)
$$

with

$$
C\left(\eta, \frac{m^{2}}{x^{2} P_{3}^{2}}, \frac{\mu^{2}}{m^{2}}\right)=\int_{0}^{1} d \xi(1-\xi)\left[\frac{1}{\sqrt{(\eta-\xi)^{2}+\frac{M^{2}}{x^{2} P_{3}^{2}}}}-\delta(\xi-\eta) \log \frac{\mu^{2}}{M^{2}}\right]
$$


Note that taking the Fourier transform, as in eq. (4.11), involves an integration of the Bessel function $K_{0}\left(z_{3} M\right)$ through its singularity at $z_{3}=0$, which is discussed in detail in appendix A. Looking at eq. (4.14), we note that, again, the coefficient $C$ contains explicit logarithms of the mass, rendering it infrared divergent. However, these divergences cancel when considering the large $P_{3}$ regime, by expanding the Fourier transform of the Bessel function in the limit $\frac{M^{2}}{\xi^{2} P_{3}^{2}} \rightarrow 0$. If $\eta>1$ or $\eta<0$, then looking at eq. (4.14) we have

$$
\lim _{P_{3} \rightarrow \infty} C\left(\eta, \frac{m^{2}}{x^{2} P_{3}^{2}}, \frac{\mu^{2}}{m^{2}}\right)=C(\eta)= \pm \int_{0}^{1} d \xi \frac{1-\xi}{\eta-\xi}= \pm\left[(1-\eta) \log \frac{\eta}{\eta-1}+1\right]
$$

where the solution with the plus refers to $\eta>1$, and the one with the minus to $\eta<0$. On the other hand, if $\eta \in(0,1)$, the factor $1 /|\eta-x|$ generated in this limit produces a non-integrable singularity at $\eta=x[22]$. To overcome this issue, as detailed in appendix A, we can write

$$
\frac{1}{\sqrt{(\eta-\xi)^{2}+\frac{M^{2}}{x^{2} P_{3}^{2}}}}=\log 4 \eta(1-\eta) \frac{x^{2} P_{3}^{2}}{M^{2}} \delta(\eta-\xi)+\frac{1}{|\eta-\xi|_{+}}+\mathcal{O}\left(\frac{M^{2}}{P_{3}^{2}}\right)
$$

so that in the region $\eta \in(0,1)$ we find

$$
\begin{aligned}
C\left(\eta, \frac{M^{2}}{x^{2} P_{3}^{2}}, \frac{\mu^{2}}{M^{2}}\right) & \stackrel{P_{3} \rightarrow \infty}{\sim} C\left(\eta, \frac{\mu^{2}}{x^{2} P_{3}^{2}}\right) \\
& =\int_{0}^{1} d \xi(1-\xi)\left[\frac{1}{|\eta-\xi|_{+}}+\delta(\eta-\xi) \log 4 \eta(1-\eta) \frac{x^{2} P_{3}^{2}}{\mu^{2}}\right]+\mathcal{O}\left(\frac{m^{2}}{P_{3}^{2}}\right) \\
& =2 \eta-1+(1-\eta) \log 4 \eta(1-\eta) \frac{x^{2} P_{3}^{2}}{\mu^{2}}+\mathcal{O}\left(\frac{m^{2}}{P_{3}^{2}}\right) .
\end{aligned}
$$

Note the cancellation of the logarithmic dependence on the mass, which leads again to a proper factorization formula, this time in momentum space. We conclude that, in momentum space, the factorization theorem is realized in the limit $P_{3} \rightarrow \infty$ and in our model this factorization theorem takes the form

$$
q\left(y, \mu^{2}, P_{3}^{2}\right)=\int_{0}^{1} \frac{d x}{x} f\left(x, \mu^{2}\right) C\left(\frac{y}{x}, \frac{\mu^{2}}{x^{2} P_{3}^{2}}\right)+\mathcal{O}\left(\frac{m^{2}}{P_{3}^{2}}\right)
$$

with

$$
C\left(\eta, \frac{\mu^{2}}{x^{2} P_{3}^{2}}\right)=\delta(1-\eta)+\alpha \begin{cases}(1-\eta) \log \frac{\eta}{\eta-1}+1 & \eta>1 \\ (1-\eta) \log 4 \eta(1-\eta) \frac{x^{2} P_{3}^{2}}{\mu^{2}}+2 \eta-1 & 0<\eta<1 \\ -(1-\eta) \log \frac{\eta}{\eta-1}-1 & \eta<0\end{cases}
$$

Factorization in position space, given in eqs. (4.5) and (4.6), is equivalent to factorization in momentum space, given in eqs. (4.18) and (4.19). In other words, taking the small- $z_{3}^{2}$ limit in position space is entirely equivalent to taking the large- $P_{3}$ limit in momentum space. This can be easily verified by computing the Fourier transfom of the small- $z_{3}^{2}$ 
coefficient $\tilde{C}$ of eq. (4.6), and checking that it is equal to the high- $P_{3}$ coefficient $C$ of eq. (4.19)

$$
\begin{aligned}
\frac{1}{x} C\left(\eta, \frac{\mu^{2}}{x^{2} P_{3}^{2}}\right) & =\frac{P_{3}}{2 \pi} \int_{-\infty}^{\infty} d z_{3} e^{-i y P_{3} z_{3}} \tilde{C}\left(x \nu, \mu^{2} z_{3}^{2}\right) \\
& =\frac{1}{x} \int_{-\infty}^{\infty} \frac{d \theta}{2 \pi} e^{-i \theta \eta} \tilde{C}\left(\theta, \frac{\mu^{2} \theta^{2}}{x^{2} P_{3}^{2}}\right) \text { with } \eta=\frac{y}{x}
\end{aligned}
$$

This check, despite being conceptually straightforward, does require some care [23]. We provide the details of the computation in appendix A. The implementation of the factorization theorem in position space, together with the definition of reduced distributions, are the typical approach followed in nonperturbative calculations of pseudo-PDFs [4, 21, 24-27], while the realization of the factorization in momentum space characterizes the quasi-PDF approach [3, 28-31].

As we have shown in this section in the simplified context of our model, these two approaches are conceptually equivalent, and related by a Fourier transform: in one case the lattice calculation needs to provide the correlators for small values of $z_{3}$, while in the other large values of $P_{3}$ are required. In both scenarios, however, the object that is actually computed is the matrix element of spatially-separated fields. This is the only quantity of interest, without the need to define either pseudo- or quasi-PDFs.

\section{$5 \quad$ Smeared distributions}

In refs. $[32,33]$, the gradient flow was proposed as an approach to control the power divergence associated with the Wilson-line operator that defines the Ioffe time distribution in QCD. The gradient flow [34-36] is a classical, gauge-invariant, one-parameter mapping of the theory that exponentially damps the UV fluctuations. This corresponds to smearing in real space, with a smearing scale that is parametrised by the flow time. In the limit of small flow time, the matrix elements of smeared fields can be related to those at vanishing flow time by a short flow-time expansion [37].

In Yang-Mills theories, gauge invariance ensures that no new divergences are introduced at finite flow time. Thus, provided the boundary theory is properly renormalized, the matrix elements of composite operators composed of fields at finite flow time are guaranteed to be finite. In the absence of gauge symmetries, the simplest method for maintaining this property is to exclude interactions from the flow time evolution of the fields, in which case this evolution corresponds to simple Gaussian smearing [38-40].

The flow time can be viewed as a non-perturbative regulator that does not affect the infrared properties of correlation functions. The smeared Ioffe-time matrix elements, constructed from fields at finite flow time, therefore satisfy the same factorization theorems as the original Ioffe-time matrix elements [32]. In the scalar case, the boundary fields $\phi(x)$ in eq. (2.1) are replaced by fields at finite flow time $\rho(t ; x)$, so that the partonic matrix element becomes

$$
\widehat{\mathcal{M}}_{t}\left(\nu, \bar{z}^{2}\right)=\langle p|\rho(t ; z) \rho(t ; 0)| p\rangle .
$$

Here the subscript indicates that the fields are evaluated at flow time $t$, and $\bar{z}^{2}=z^{2} / t$. 
The gradient flow is only well-defined in Euclidean space, but for $z^{2}<0$, the matrix elements are signature independent [41]. The tree-level and one-loop diagrams that contribute to this matrix element are exactly those given in figure 2, with $\phi(x)$ replaced by $\rho(t ; x)$. Working in the small flow-time regime, where contributions of $\mathcal{O}(t)$ can be neglected, the only diagram that must be calculated is diagram (c) of figure 2 . Therefore, we can deduce the factorization properties of the smeared matrix element directly from the analogue of eq. (2.11) at nonzero flow time

$$
\begin{aligned}
\widehat{\mathcal{M}}_{t}^{(1)}\left(\nu,-\bar{z}_{3}^{2}\right) & =g^{2} \int_{k_{E}} e^{-2 k_{E}^{2} t} \frac{e^{-i k_{\mathrm{E}} z_{3}}}{\left(k_{\mathrm{E}}^{2}+m^{2}\right)^{2}} \frac{1}{\left(p_{\mathrm{E}}-k_{\mathrm{E}}\right)^{2}+m^{2}} \\
& =g^{2} \int_{0}^{1} d \xi(1-\xi) K_{t}\left(-\bar{z}_{3}^{2}, \bar{M}^{2}\right) \widehat{\mathcal{M}}^{(0)}(\xi \nu, 0),
\end{aligned}
$$

where the exponential damping is the result of the smearing of the fields and we have introduced $\bar{M}^{2}=M^{2} t$. Here the kernel $K_{t}\left(-\bar{z}_{3}^{2}, \bar{M}^{2}\right)$ is given by

$$
K_{t}\left(-\bar{z}_{3}^{2}, \bar{M}^{2}\right)=\frac{\mu^{6-d}}{(4 \pi)^{d / 2}} e^{-2 m^{2} t \xi} \int_{0}^{\infty} \mathrm{d} T \frac{T^{2}}{(T+2 t)^{d / 2}} e^{-T M^{2}} e^{\left(4 \xi t p_{\mathrm{E}}-i z_{\mathrm{E}}\right)^{2} /(4(T+2 t))},
$$

which reduces to the kernel in eq. (2.16) when $t=0$.

By introducing the further dimensionless variables $\bar{\mu}^{2}=\mu^{2} t, \bar{m}^{2}=m^{2} t$, and

$$
\beta^{2}=-\frac{1}{t}\left(\xi t p_{\mathrm{E}}^{\mu}-\frac{i z_{\mathrm{E}}^{\mu}}{2}\right)^{2}=\xi^{2} \bar{m}^{2}+i \xi \nu+\frac{\bar{z}_{3}^{2}}{4}
$$

and changing variables to $u=T / t+2$, the integral becomes

$$
K_{t}\left(-\bar{z}_{3}^{2}, \bar{M}^{2}\right)=\frac{\bar{\mu}^{6-d}}{(4 \pi)^{d / 2}} e^{-2(\xi-1)^{2} \bar{m}^{2}} \int_{2}^{\infty} \mathrm{d} u \frac{(u-2)^{2}}{u^{d / 2}} e^{-u \bar{M}^{2}-\beta^{2} / u} .
$$

This integral can be solved in terms of incomplete Bessel functions [42-44], which can be studied in various asymptotic regimes. In particular,

$$
\begin{aligned}
K_{t}\left(-\bar{z}_{3}^{2}, \bar{M}^{2}\right)= & \frac{2 \bar{\mu}^{6-d}}{(4 \pi)^{d / 2}} e^{-2 \frac{(\xi-1)^{2}}{1-\xi+\xi^{2}} \bar{M}^{2}} \\
& \times\left[K_{0}(2|\bar{M} \beta|, 2)-4 \frac{\bar{M}}{|\beta|} K_{1}(2|\bar{M} \beta|, 2)+4 \frac{\bar{M}^{2}}{\beta^{2}} K_{2}(2|\bar{M} \beta|, 2)\right]
\end{aligned}
$$

where

$$
K_{n}(y, a)=K_{n}(y)-J(y, n, a),
$$

with $J(y, n, a)$ the finite integral

$$
J(y, n, a)=\int_{0}^{a} \mathrm{~d} v e^{-y \cosh (v)} \cosh (n v) .
$$

This result is finite in six dimensions, because the incomplete Bessel functions are finite at finite flow time and quark mass. Indeed, one can evaluate these integrals numerically 
by imposing a cutoff. For sufficiently large cutoff, the results are independent of the cutoff value. Using eq. (5.7), eq. (5.6) can be written as

$$
\begin{aligned}
K_{t}\left(-\bar{z}_{3}^{2}, \bar{M}^{2}\right)= & \frac{2}{(4 \pi)^{3}} e^{-2 \frac{(\xi-1)^{2}}{1-\xi+\xi^{2}} \bar{M}^{2}}\left\{K_{0}(2|\bar{M} \beta|)-4 \frac{\bar{M}}{|\beta|} K_{1}(2|\bar{M} \beta|)+4 \frac{\bar{M}^{2}}{\beta^{2}} K_{2}(2|\bar{M} \beta|)\right. \\
& \left.-J(2|\bar{M} \beta|, 0,2)+4 \frac{\bar{M}}{|\beta|} J(2|\bar{M} \beta|, 1,2)-4 \frac{\bar{M}^{2}}{\beta^{2}} J(2|\bar{M} \beta|, 2,2)\right\} .
\end{aligned}
$$

In the limit where

$$
\frac{t^{2} m^{2}}{z_{E}^{2}} \ll 1
$$

the argument of the Bessel functions, $|\bar{M} \beta|$, can be expressed as

$$
2|\bar{M} \beta|=M\left|z_{E}\right|+\mathcal{O}\left(\frac{t^{2} m^{2}}{z_{E}^{2}}\right)=M z_{3}+\mathcal{O}\left(\frac{t^{2} m^{2}}{z_{E}^{2}}\right),
$$

so that, in the limit of small $z_{3}$ we can expand them as

$$
\begin{aligned}
2 K_{0}(2|\bar{M} \beta|) & =-\log \left(M^{2} z_{3}^{2}\right)+2 \log \left(2 e^{-\gamma_{E}}\right)+\mathcal{O}\left(m^{2} z_{3}^{2}, \frac{t^{2} m^{2}}{z_{E}^{2}}\right) \\
2 \frac{\bar{M}}{|\beta|} K_{1}(2|\bar{M} \beta|) & =0+\mathcal{O}\left(m^{2} z_{3}^{2}, \frac{t^{2} m^{2}}{z_{E}^{2}}, 1 / \bar{z}^{2}\right) \\
2 \frac{\bar{M}^{2}}{\beta^{2}} K_{2}(2|\bar{M} \beta|) & =0+\mathcal{O}\left(m^{2} z_{3}^{2}, \frac{t^{2} m^{2}}{z_{E}^{2}}, 1 / \bar{z}^{2}\right) .
\end{aligned}
$$

Care must be taken when matching these expressions to the light-cone case. The limits need to be taken in the right order so that the quantity $\frac{t^{2} m^{2}}{z_{E}^{2}}$ remains small in the process. One must first consider the small flow time regime at fixed $z_{3}$, in which $\bar{z} \gg 1$, and then consider the limit in which $m^{2} z_{3}^{2}$ goes to zero. Taking the limit of small $m^{2} z_{3}^{2}$ at fixed $t$ would violate the condition above and invalidate the factorization theorem, viz. data for values of $t$ and $z_{3}$ that correspond to large values of $t^{2} m^{2} / z_{E}^{2}$ are not described by the factorization theorems discussed here. With this in mind, the only logarithmic infrared divergence occurs in the first Bessel function, which has been expanded using eq. (4.4). Thus, in the small flow-time regime eq. (5.9) becomes

$$
\begin{aligned}
K_{t}\left(-\bar{z}_{3}^{2}, \bar{M}^{2}\right)= & \frac{1}{(4 \pi)^{3}}\left[-\log \left(M^{2} z_{3}^{2}\right)+2 \log \left(2 e^{-\gamma_{E}}\right)+\mathcal{R}\left(M z_{3}\right)\right] \\
& +\mathcal{O}\left(m^{2} z_{3}^{2}, \frac{t^{2} m^{2}}{z_{E}^{2}}, 1 / \bar{z}^{2}\right)
\end{aligned}
$$

where the rational function $\mathcal{R}\left(M z_{3}\right)$ contains the IR finite contributions generated by the $J$ functions of eq. (5.8). The logarithmic IR divergence in eq. (5.15), regularized by the mass $m$, matches those in eqs. (2.21) and (3.6).

In the short flow-time regime, the one-loop contributions to eq. (5.1) from diagrams (a) and (b) are just those given in eq. (2.9). The corresponding renormalized quantity at 
one loop is therefore

$$
\begin{aligned}
\widehat{\mathcal{M}}_{t}\left(\nu,-z_{3}^{2} ; \mu^{2}\right)= & {\left[1+\alpha\left(\frac{1}{6} \log \frac{m^{2}}{\mu^{2}}+b\right)\right] \widehat{\mathcal{M}}^{(0)}(\nu, 0) } \\
& +\alpha \int_{0}^{1} d \xi(1-\xi)\left[-\log \left(M^{2} z_{3}^{2}\right)+2 \log \left(2 e^{-\gamma_{E}}\right)+\mathcal{R}\left(M z_{3}\right)\right] \widehat{\mathcal{M}}^{(0)}(x \nu, 0) .
\end{aligned}
$$

We can now directly relate this quantity, via a factorization relation, to the light-cone quantity $f\left(x, \mu^{2}\right)$ using eq. (2.25). We obtain

$$
\widehat{\mathcal{M}}_{t}\left(\nu,-z_{3}^{2} ; \mu^{2}\right)=\int_{0}^{1} d x \bar{C}\left(x \nu, \mu^{2} z_{3}^{2}\right) \widehat{f}\left(x, \mu^{2}\right)
$$

with

$$
\begin{aligned}
\bar{C}\left(x \nu, \mu^{2} z_{3}^{2}\right)= & e^{i x \nu}-\alpha \int_{0}^{1} d \xi(1-\xi)\left[\log \left(\mu^{2} z_{3}^{2} \frac{e^{2 \gamma_{E}}}{4}\right)-\mathcal{R}\left(M z_{3}\right)\right] e^{i \xi x \nu} \\
& +\mathcal{O}\left(m^{2} z_{3}^{2}, \frac{t^{2} m^{2}}{z_{E}^{2}}, 1 / \bar{z}^{2}\right)
\end{aligned}
$$

This factorization relation provides the explicit connection between the collinear PDFs and an equal-time correlator at nonzero flow time, through a convolution with a perturbative kernel.

\section{Conclusions}

We have addressed the definition and renormalization of equal-time correlators whose computation can be performed on the lattice, studying their relation with the corresponding light-cone matrix elements underlying the definition of collinear PDFs via factorization theorems. To highlight and clarify the most important aspects of the factorization theorems, we have studied them in the context of a nongauge theory. This allows us to avoid the formal complications that arise in QCD, which can obscure the key concepts. We derive the relation between the light-cone and Euclidean matrix elements at the one-loop level, and then study the limits that lead to well-defined factorization theorems. These relations express suitable correlators that are evaluated by Monte Carlo calculations in terms of a convolution between a collinear PDF and an infrared safe coefficient function, which can be evaluated in perturbation theory. We obtain factorization theorems in both position and momentum space, by considering the regimes of small- $z_{3}^{2}$ and large- $P_{3}$ respectively, and show that these limits are equivalent at one loop, which highlights the formal equivalence of the pseudo- and quasi-PDFs approach. In addition, we demonstrate that the gradient flow can be used to define a new class of lattice observables that satisfy factorization.

These ideas naturally suggest that the lattice data should be used in a fitting framework to extract PDFs, in the same way experimental data are usually included in global QCD analyses. This approach has been studied at NLO in $[17,45]$ and is in the spirit of the "good lattice cross-sections" (or factorizable matrix elements) proposed in refs. [46, 47]. Results for the NNLO coefficients entering the factorization theorem have recently become available in both position and momentum space [48-52], paving the way to NNLO fits. 
The general idea is straightforward: the unknown $x$-dependence of the PDF at a specific fitting scale is parametrized by introducing a suitable functional form. The PDF at a generic scale can be computed in terms of its parametric form at the fitting scale, which then leads to a theoretical prediction for the lattice observable when working in either the small$z_{3}^{2}$ or large- $P_{3}$ limit. Assuming that we have a set of lattice results for the real and imaginary part of the Ioffe-time matrix elements, a standard minimum- $\chi^{2}$ fit yields the values of the free parameters that best describe such data. As in any other PDF determination, we highlight the importance of having a robust estimate of the full covariance matrix that enters the $\chi^{2}$ definition, and this should be provided by the lattice group performing the calculation.

We also stress that this procedure is exactly the one that is currently used to extract PDFs from experimental data [53-57], with the lattice matrix elements playing the same role as the cross-sections for high-energy processes. Given a discrete set of points for quantities that are connected to collinear PDFs through a factorization theorem, we can use them to perform a fit, thereby obtaining an estimate of the PDFs and their corresponding error.

In this work we demonstrate, at one loop in a scalar model, the conceptual equivalence of the pseudo and quasi distribution methods, and advocated for a fitting framework that directly relates Ioffe time distributions to light-cone PDFs. We emphasize, however, that conceptual equivalence may not translate to equivalence in practice. On the one hand, the LaMET approach relies on large hadronic momenta to suppress higher twist contamination. On the other hand, the pseudo distribution approach uses small spatial separations to suppress higher twist effects, but requires large momenta to cover a range of Ioffe times. In both cases, large values of the hadron momentum can lead to significant signal-to-noise challenges and discretization effects of the form $(a P)^{n}$. The interplay of higher twist contamination and discretization effects is nontrivial and will depend both on the details of the distribution itself and on the specific choice of discretization. These effects must be studied systematically, across a wide range of observables, to pin down systematic uncertainties and strengthen the role that lattice QCD can play in the determination of hadron structure.

\section{Acknowledgments}

We are thankful to K. Orginos, E.R. Nocera, A.V. Radyushkin, G.C. Rossi, and M. Testa for useful discussions.

TG is supported by The Scottish Funding Council, grant H14027. C.J.M. is supported in part by the U.S. Department of Energy, Office of Science, Office of Nuclear Physics under contract No. DE-AC05-06OR23177. LDD is supported by an STFC Consolidated Grant, ST/P0000630/1, and a Royal Society Wolfson Research Merit Award, WM140078.

\section{A Momentum space factorization}

In this appendix we report in detail some of the computations performed in section 4.2 , to obtain the coefficient $C$ of eq. (4.14) and its high momentum limit of eq. (4.19). In order to compute the Fourier transform of the coefficient $\tilde{C}$ entering eq. (4.2), we perform a change 
variable, $\theta=\xi P_{3} z_{3}$, and define $\eta=\frac{y}{\xi}$, so that

$$
\begin{aligned}
& \frac{P_{3}}{2 \pi} \int_{-\infty}^{\infty} d z_{3} e^{-i y P_{3} z_{3}} \tilde{C}\left(x P_{3} z_{3}, m z_{3}, \frac{\mu^{2}}{m^{2}}\right)=\frac{1}{x} \int_{-\infty}^{\infty} \frac{d \theta}{2 \pi} e^{-i \eta \theta} \tilde{C}\left(\theta, \frac{m \theta}{x P_{3}}, \frac{\mu^{2}}{m^{2}}\right)= \\
& \quad=\frac{1}{x}\left[\delta(\eta-1)-\alpha \int_{0}^{1} d \xi(1-\xi) \int_{-\infty}^{\infty} \frac{d \theta}{2 \pi} e^{-i(\eta-\xi) \theta}\left(2 K_{0}\left(\frac{M \theta}{x P_{3}}\right)-\log \frac{\mu^{2}}{M^{2}}\right)\right] .
\end{aligned}
$$

The Fourier transform of the Bessel function, obtained also in ref. [58], can be computed using the integral representation in eq. (3.1), computing the gaussian integral over $\theta$ first:

$$
\int_{-\infty}^{\infty} \frac{d \theta}{2 \pi} e^{-i(\eta-\xi) \theta} \int_{0}^{\infty} \frac{d T}{T} e^{-T} e^{-\left(\frac{M \theta}{x P_{3}}\right)^{2} \frac{1}{4 T}}=\frac{1}{\sqrt{(\eta-\xi)^{2}+\frac{M^{2}}{x^{2} P_{3}^{2}}}}
$$

so that the $\mathcal{O}(\alpha)$ contribution to (A.1) can be written as

$$
\int_{0}^{1} d \xi(1-\xi)\left[\frac{1}{\sqrt{(\eta-\xi)^{2}+\frac{M^{2}}{x^{2} P_{3}^{2}}}}-\delta(\xi-\eta) \log \frac{\mu^{2}}{M^{2}}\right] .
$$

As mentioned in section 4.2 , the computation of the large- $P_{3}$ limit when $\eta \in(0,1)$ requires additional care, since the integrand develops a non-integrable divergence for $\xi=\eta$ when $M^{2} /\left(x^{2} P_{3}^{2}\right) \rightarrow 0$. This issue was first addressed and solved in ref. [22] in the context of QCD. Since in the scalar theory the same kind of Bessel function appears, its Fourier transform leads to an analogous singularity. In order to elucidate this problem, given a generic test function $\phi(\xi)$, we consider the integral

$$
\int_{0}^{1} d \xi \frac{\phi(\xi)}{\sqrt{(\eta-\xi)^{2}+\kappa^{2}}}
$$

in the limit where $\kappa \rightarrow 0$. Defining

$$
G\left(\eta, \kappa^{2}\right)=\int_{0}^{1} \frac{d \xi}{\sqrt{(\xi-\eta)^{2}+\kappa^{2}}}
$$

allows us to rewrite eq. A.4 above as

$$
\int_{0}^{1} d \xi \frac{\phi(\xi)}{\sqrt{(\eta-\xi)^{2}+\kappa^{2}}}=\phi(\eta) G\left(\eta, \kappa^{2}\right)+\int_{0}^{1} d \xi \frac{1}{\sqrt{(\eta-\xi)^{2}+\kappa^{2}}}(\phi(\xi)-\phi(\eta)) .
$$

The divergence of the original integral is encoded in the function $G\left(\eta, \kappa^{2}\right)$, which can be readily evaluated:

$$
G\left(\eta, \kappa^{2}\right)=\log \left(4 \eta(1-\eta) \frac{1}{\kappa^{2}}\right)+\mathcal{O}\left(\kappa^{2}\right)
$$

The integral on the r.h.s. of (A.6) is convergent for $\kappa \rightarrow 0$, and we have

$$
\begin{aligned}
\int_{0}^{1} d \xi \frac{1}{\sqrt{(\eta-\xi)^{2}+\kappa^{2}}}(\phi(\xi)-\phi(\eta)) & =\int_{0}^{1} d \xi \frac{1}{|\xi-\eta|}(\phi(\xi)-\phi(\eta))+\mathcal{O}\left(\kappa^{2}\right) \\
& =\int_{0}^{1} d \xi \frac{1}{|\xi-\eta|_{+}} \phi(\xi)+\mathcal{O}\left(\kappa^{2}\right)
\end{aligned}
$$


Therefore, collecting both contributions,

$$
\frac{1}{\sqrt{(\eta-\xi)^{2}+\kappa^{2}}}=\delta(\eta-\xi) \log \left(4 \eta(1-\eta) \frac{1}{\kappa^{2}}\right)+\frac{1}{|\eta-\xi|_{+}}+\mathcal{O}\left(\kappa^{2}\right) .
$$

\section{B Equivalence between pseudo- and quasi-PDF approaches}

As discussed at the end of section 4 , taking the small- $z_{3}^{2}$ limit in position space is equivalent to taking the large- $P_{3}$ limit in momentum space. This can be verified at 1-loop by showing that the coefficent functions of eqs. (4.6) and (4.19) are related through a Fourier transform, as stated in eq. (4.20). Here we report the details of the computation. Taking the Fourier transform of the small- $z_{3}^{2}$ coefficient of eq. (4.6) and defining $\eta=y / x$ we have

$$
\begin{aligned}
\frac{P_{3}}{2 \pi} \int_{-\infty}^{\infty} d z_{3} e^{-i y P_{3} z_{3}} \tilde{C}\left(x \nu, \mu^{2} z_{3}^{2}\right)= & \frac{1}{x} \int_{-\infty}^{\infty} \frac{d \theta}{2 \pi} e^{-i \theta \eta} \tilde{C}\left(\theta, \frac{\mu^{2} \theta^{2}}{x^{2} P_{3}^{2}}\right) \\
=\frac{1}{x} & {\left[\delta(\eta-1)+\alpha \log \frac{4\left(x P_{3}\right)^{2}}{\mu^{2} e^{2 \gamma_{E}}} \int_{0}^{1} d \xi \delta(\xi-\eta)(1-\xi)\right.} \\
& \left.-\alpha \int_{0}^{1} d \xi(1-\xi) \int_{-\infty}^{\infty} \frac{d \theta}{2 \pi} e^{-i(\eta-\xi) \theta} \log \theta^{2}\right] .
\end{aligned}
$$

Following ref. [23], the Fourier transform of $\log \theta^{2}$ can be defined as

$$
\begin{aligned}
\int \frac{d \theta}{2 \pi} e^{-i t \theta} \log \theta^{2} & =\left[\frac{d}{d \tau} \int \frac{d \theta}{2 \pi} e^{-i t \theta}\left(\theta^{2}\right)^{\tau}\right]_{\tau=0} \\
& =-2 \gamma_{E} \delta(t)-\frac{\theta(1-|t|)}{|t|_{(+0)}}-\frac{\theta(|t|-1)}{|t|_{(+\infty)}}+\frac{1}{(t)^{2}} \delta\left(\frac{1}{|t|}\right),
\end{aligned}
$$

with

$$
\begin{aligned}
\frac{1}{|t|}_{(+0)} & =\lim _{a \rightarrow 0}\left[\frac{\theta(|t|-a)}{|t|}+\delta(|t|-a) \log a\right], \\
\frac{1}{|t|}(+\infty) & =\frac{1}{(t)^{2}} \lim _{a \rightarrow 0}\left[\theta\left(\frac{1}{|t|}-a\right)|t|+\delta\left(\frac{1}{|t|}-a\right) \log a\right], \\
\delta\left(\frac{1}{|t|}\right) & =\lim _{a \rightarrow 0} \delta\left(\frac{1}{|t|}-a\right) .
\end{aligned}
$$

The proof of eq. (B.2) can be found, for example, in the appendix A and C of ref. [23], to which we refer for more details. Setting $t=\eta-\xi$ and plugging everything in eq. (B.1), remembering that $\xi \in[0,1]$, we get different answers depending on the value of $\eta$. For $\eta \in[0,1]$, just the first two terms in eq. (B.2) contribute, giving

$$
\begin{gathered}
\int_{0}^{1} d \xi\left[2 \gamma_{E} \delta(\eta-\xi)-\lim _{a \rightarrow 0}\left(\frac{\theta(|\eta-\xi|-\beta)}{|\eta-\xi|}+\delta(|\eta-\xi|-a) \log a\right)\right](1-\xi) \\
=\log e^{2 \gamma_{E}}(1-\eta)+(1-\eta) \log \eta(1-\eta)+2 \eta-1,
\end{gathered}
$$

while for $\eta>1$ or $\eta<0$ the third contribution in eq. (B.2) gives simply

$$
-\int_{0}^{1} d \xi(1-\xi) \frac{|\eta-\xi|}{(\eta-\xi)^{2}}
$$


Looking at the last term in eq. (B.2), considering its contribution to the convolution integral with the PDF and doing the integral over $x$ first we find

$$
\lim _{a \rightarrow 0} \int_{0}^{1} \frac{d x}{x} \int_{0}^{1} d \xi(1-\xi) \delta\left(\frac{1}{\left|\frac{y}{x}-\xi\right|}-a\right) f(x) \propto \lim _{a \rightarrow 0} a^{2} f(a)=0 .
$$

Using eqs. (B.6), (B.7), (B.8) in eq. (B.1) we find back the expression for $C\left(\eta, \frac{\mu^{2}}{x^{2} P_{3}^{2}}\right)$ as in eq. (4.19), which completes our check.

\section{Quasi-PDFs and their moments}

As mentioned in the introduction of this paper, the works where the concept of quasi-PDF was first introduced have been criticized in refs. [11, 12], where it was argued that such approach does not give access to the full nonperturbative PDF. In support of their argument, the Authors have shown that moments of quasi-PDFs are divergent: since the moments of parton distributions should reproduce the (finite) matrix elements of the renormalized local DIS operator, they conclude that the quasi-PDF cannot be considered as an euclidean generalization of the light-cone PDF. The problem has been addressed in several independent papers, see e.g. refs. $[13,14,59]$. In this appendix we revise these criticisms in the framework of the scalar model: first we show how the points raised in refs. [11, 12] can be easily seen and understood within the toy model presented in this paper, showing explicitly how all the moments of quasi-PDFs are indeed divergent; second we discuss how such feature does not invalidate the programme presented in section 6 , based on the determination of a parametric form of the light-cone PDF based on a discrete set of data for the euclidean matrix element.

We start this section by computing the moments of the quasi-PDF. From eq. (3.2), using the integral representation of the Bessel function, the $\mathcal{O}(\alpha)$ contribution to the euclidean matrix element reads

$$
\hat{\mathcal{M}}^{(1)}\left(\nu,-z_{3}^{2}\right)=\alpha \int_{0}^{1} d \xi(1-\xi) \int_{0}^{\infty} \frac{d T}{T} e^{-T} e^{-\frac{z_{3}^{2} M^{2}}{4 T}} e^{-i \xi P_{3} z_{3}} .
$$

The corresponding contribution to the quasi-PDF is found by taking the Fourier transform of the expression above:

$$
\begin{aligned}
\hat{q}^{(1)}(y) & =\frac{P_{3}}{2 \pi} \int_{-\infty}^{\infty} d z_{3} e^{-i y P_{3} z_{3}} \hat{\mathcal{M}}^{(1)}\left(\nu,-z_{3}^{2}\right) \\
& =\alpha \frac{P_{3}}{\sqrt{\pi}} \int_{0}^{1} d \xi(1-\xi) \frac{1}{M} \int_{0}^{\infty} \frac{d T}{\sqrt{T}} e^{-T} e^{-T(y+\xi)^{2}} \frac{P_{3}^{2}}{M^{2}}
\end{aligned}
$$

where in the last line we have computed the gaussian integral over $z_{3}$. Taking the $n$-th moment of $\hat{q}^{(1)}(y)$ yields

$$
\int_{-\infty}^{\infty} d y y^{n} \hat{q}^{(1)}(y)=\alpha \frac{P_{3}}{\sqrt{\pi}} \int_{0}^{1} d \xi(1-\xi) \frac{1}{M} \int_{0}^{\infty} \frac{d T}{\sqrt{T}} e^{-T} \int_{-\infty}^{\infty} d y(y-\xi)^{n} e^{-T y^{2} \frac{P_{3}^{2}}{M^{2}}}
$$


We can expand the polynomial term as

$$
(y-\xi)^{n}=\sum_{k=0}^{n}\left(\begin{array}{l}
k \\
n
\end{array}\right) y^{n-k} \xi^{k}
$$

and evaluate each contribution in turn. The term with $k=n$, performing the integral over $y$ first, yields

$$
\begin{aligned}
\alpha \frac{P_{3}}{\sqrt{\pi}} & \int_{0}^{1} d \xi(1-\xi) \xi^{n} \frac{1}{M} \int_{0}^{\infty} \frac{d T}{\sqrt{T}} e^{-T} \int_{-\infty}^{\infty} d y e^{-T y^{2} \frac{P_{3}^{2}}{M^{2}}} \\
& =\alpha \int_{0}^{1} d \xi(1-\xi) \xi^{n} \int_{0}^{\infty} \frac{d T}{T} e^{-T} .
\end{aligned}
$$

The integral over $T$ is divergent, with the divergence originating from the lower end of the integration region, i.e. when $T \rightarrow 0$. Introducing a cutoff $a^{2}$ for small values of $T^{3}$ and considering the limit $a^{2} \rightarrow 0$, we get the logarithmic divergent contribution

$$
\alpha \int_{0}^{1} d \xi(1-\xi) \xi^{n} \int_{a^{2}}^{\infty} \frac{d T}{T} e^{-T} \stackrel{a^{2} \rightarrow 0}{\sim}-\alpha \int_{0}^{1} d \xi(1-\xi) \xi^{n} \log a^{2}
$$

Similarly we can consider contributions coming from even values of $n-k$. Using

$$
\begin{gathered}
\int_{-\infty}^{\infty} d y y^{2 m} e^{-T y^{2} \frac{P_{3}^{2}}{M^{2}}}=\frac{M}{P_{3}}\left(-\frac{M^{2}}{P_{3}^{2}} \frac{d}{d T}\right)^{m} \int_{-\infty}^{\infty} d y e^{-T y^{2}} \\
=\frac{M \sqrt{\pi}}{P_{3}}\left(-\frac{M^{2}}{P_{3}^{2}} \frac{d}{d T}\right)^{m} \frac{1}{\sqrt{T}} \propto \frac{M \sqrt{\pi}}{P_{3}} \frac{1}{T^{m+\frac{1}{2}}}
\end{gathered}
$$

and considering $n-k=2 m$, we get

$$
\begin{aligned}
\alpha \frac{P_{3}}{\sqrt{\pi}} & \int_{0}^{1} d \xi(1-\xi) \xi^{n-2 m} \frac{1}{M} \int_{0}^{\infty} \frac{d T}{\sqrt{T}} e^{-T} \int_{-\infty}^{\infty} d y y^{2 m} e^{-T y^{2} \frac{P_{3}^{2}}{M^{2}}} \\
& \propto \alpha \int_{0}^{1} d \xi(1-\xi) \xi^{n-2 m} \int_{a^{2}}^{\infty} \frac{d T}{T^{m+1}} e^{-T} \stackrel{a^{2} \rightarrow 0}{\sim} \alpha \int_{0}^{1} d \xi(1-\xi) \xi^{n-2 m} \frac{1}{m}\left(\frac{1}{a^{2}}\right)^{m},
\end{aligned}
$$

where again we have introduced a cutoff $a^{2}$ for small values of $T$ and considered the limit $a^{2} \rightarrow 0$. Contributions from odd values of $n-k$ vanish. Looking at eqs. (C.8), (C.10) it is then clear that all the moments of the quasi-PDFs will be at least logarithmically divergent with the cutoff $a^{2}$, with higher moments affected by higher power divergences.

This relatively simple calculation shows that we obtain divergent contributions for the moments of the quasi-PDF and therefore quasi-PDFs cannot be considered as the proper euclidean generalization of the light-cone parton distribution. This, however, does not invalidate the approach described in section 6: as mentioned, what really matters is the existence of a factorization theorem connecting the collinear PDF with a renormalizable quantity that can be computed on the lattice, which in our case will be the euclidean matrix element of eq. (3.6), computed for fixed values of $P_{3}$ and $z_{3}$. As long as $z_{3}$ is kept

\footnotetext{
${ }^{3}$ The cutoff $a$ has dimensions of length and can be thought of as a lattice spacing if the theory were regulated on a lattice.
} 
small and different from 0, the factorization formula (4.5) holds, and can be used to fit the light-cone PDF using the available lattice data. How well such data can constrain the PDF is something which should be investigated, just as in the same way the constraints from new experimental measurements are usually analyzed.

Open Access. This article is distributed under the terms of the Creative Commons Attribution License (CC-BY 4.0), which permits any use, distribution and reproduction in any medium, provided the original author(s) and source are credited.

\section{References}

[1] X. Ji, Parton physics from large-momentum effective field theory, Sci. China Phys. Mech. Astron. 57 (2014) 1407 [arXiv: 1404.6680] [INSPIRE].

[2] X. Ji, Y.-S. Liu, Y. Liu, J.-H. Zhang and Y. Zhao, Large-momentum effective theory, arXiv:2004.03543 [INSPIRE].

[3] X. Ji, Parton physics on a Euclidean lattice, Phys. Rev. Lett. 110 (2013) 262002 [arXiv: 1305.1539] [INSPIRE].

[4] A.V. Radyushkin, Quasi-parton distribution functions, momentum distributions, and pseudo-parton distribution functions, Phys. Rev. D 96 (2017) 034025 [arXiv:1705.01488] [INSPIRE].

[5] L. Del Debbio, Parton distributions in the LHC era, EPJ Web Conf. 175 (2018) 01006 [INSPIRE].

[6] C. Monahan, Recent developments in x-dependent structure calculations, PoS (LATTICE2018) 018 (2018) [arXiv: 1811.00678] [INSPIRE].

[7] Y. Zhao, Unraveling high-energy hadron structures with lattice QCD, Int. J. Mod. Phys. A 33 (2019) 1830033 [arXiv: 1812.07192] [INSPIRE].

[8] K. Cichy and M. Constantinou, A guide to light-cone PDFs from lattice QCD: an overview of approaches, techniques and results, Adv. High Energy Phys. 2019 (2019) 3036904 [arXiv: 1811.07248] [INSPIRE].

[9] A.V. Radyushkin, Theory and applications of parton pseudodistributions, Int. J. Mod. Phys. A 35 (2020) 2030002 [arXiv: 1912.04244] [INSPIRE].

[10] M. Constantinou et al., Parton distributions and lattice QCD calculations: toward $3 D$ structure, arXiv:2006.08636 [INSPIRE].

[11] G.C. Rossi and M. Testa, Note on lattice regularization and equal-time correlators for parton distribution functions, Phys. Rev. D 96 (2017) 014507 [arXiv: 1706. 04428] [InSPIRE].

[12] G. Rossi and M. Testa, Euclidean versus Minkowski short distance, Phys. Rev. D 98 (2018) 054028 [arXiv: 1806.00808] [INSPIRE].

[13] A.V. Radyushkin, Structure of parton quasi-distributions and their moments, Phys. Lett. B 788 (2019) 380 [arXiv: 1807.07509] [INSPIRE].

[14] J. Karpie, K. Orginos and S. Zafeiropoulos, Moments of Ioffe time parton distribution functions from non-local matrix elements, JHEP 11 (2018) 178 [arXiv:1807.10933] [INSPIRE]. 
[15] J.C. Collins, Intrinsic transverse momentum. 1. Nongauge theories, Phys. Rev. D 21 (1980) 2962 [INSPIRE].

[16] J.C. Collins and D.E. Soper, Parton distribution and decay functions, Nucl. Phys. B 194 (1982) 445 [INSPIRE].

[17] K. Cichy, L. Del Debbio and T. Giani, Parton distributions from lattice data: the nonsinglet case, JHEP 10 (2019) 137 [arXiv: 1907.06037] [INSPIRE].

[18] T. Ishikawa, Y.-Q. Ma, J.-W. Qiu and S. Yoshida, Renormalizability of quasiparton distribution functions, Phys. Rev. D 96 (2017) 094019 [arXiv: 1707.03107] [InSPIRE].

[19] J.-H. Zhang, J.-W. Chen and C. Monahan, Parton distribution functions from reduced Ioffe-time distributions, Phys. Rev. D 97 (2018) 074508 [arXiv:1801.03023] [InSPIRE].

[20] A. Radyushkin, One-loop evolution of parton pseudo-distribution functions on the lattice, Phys. Rev. D 98 (2018) 014019 [arXiv:1801.02427] [INSPIRE].

[21] K. Orginos, A. Radyushkin, J. Karpie and S. Zafeiropoulos, Lattice QCD exploration of parton pseudo-distribution functions, Phys. Rev. D 96 (2017) 094503 [arXiv:1706.05373] [INSPIRE].

[22] A.V. Radyushkin, Quark pseudodistributions at short distances, Phys. Lett. B 781 (2018) 433 [arXiv: 1710.08813] [INSPIRE].

[23] T. Izubuchi, X. Ji, L. Jin, I.W. Stewart and Y. Zhao, Factorization theorem relating Euclidean and light-cone parton distributions, Phys. Rev. D 98 (2018) 056004 [arXiv:1801.03917] [INSPIRE].

[24] B. Joó, J. Karpie, K. Orginos, A. Radyushkin, D. Richards and S. Zafeiropoulos, Parton distribution functions from Ioffe time pseudo-distributions, JHEP 12 (2019) 081 [arXiv: 1908.09771] [INSPIRE].

[25] B. Joó et al., Pion valence structure from Ioffe-time parton pseudodistribution functions, Phys. Rev. D 100 (2019) 114512 [arXiv:1909.08517] [INSPIRE].

[26] B. Joó, J. Karpie, K. Orginos, A.V. Radyushkin, D.G. Richards and S. Zafeiropoulos, Parton distribution functions from Ioffe time pseudo-distributions from lattice calculations; approaching the physical point, arXiv:2004.01687 [INSPIRE].

[27] A.V. Radyushkin, Generalized parton distributions and pseudodistributions, Phys. Rev. D 100 (2019) 116011 [arXiv:1909.08474] [INSPIRE].

[28] C. Alexandrou, K. Cichy, M. Constantinou, K. Jansen, A. Scapellato and F. Steffens, Light-cone parton distribution functions from lattice QCD, Phys. Rev. Lett. 121 (2018) 112001 [arXiv: 1803.02685] [INSPIRE].

[29] C. Alexandrou et al., Systematic uncertainties in parton distribution functions from lattice QCD simulations at the physical point, Phys. Rev. D 99 (2019) 114504 [arXiv:1902.00587] [INSPIRE].

[30] Y. Chai et al., Parton distribution functions of $\Delta^{+}$on the lattice, Phys. Rev. D 102 (2020) 014508 [arXiv: 2002 .12044] [INSPIRE].

[31] M. Bhat, K. Cichy, M. Constantinou and A. Scapellato, Parton distribution functions from lattice QCD at physical quark masses via the pseudo-distribution approach, arXiv: 2005.02102 [INSPIRE]. 
[32] C. Monahan and K. Orginos, Quasi parton distributions and the gradient flow, JHEP 03 (2017) 116 [arXiv : 1612.01584] [INSPIRE].

[33] C. Monahan, Smeared quasidistributions in perturbation theory, Phys. Rev. D 97 (2018) 054507 [arXiv: 1710.04607] [INSPIRE].

[34] R. Narayanan and H. Neuberger, Infinite $N$ phase transitions in continuum Wilson loop operators, JHEP 03 (2006) 064 [hep-th/0601210] [INSPIRE].

[35] M. Lüscher and P. Weisz, Perturbative analysis of the gradient flow in non-Abelian gauge theories, JHEP 02 (2011) 051 [arXiv:1101.0963] [INSPIRE].

[36] M. Lüscher, Chiral symmetry and the Yang-Mills gradient flow, JHEP 04 (2013) 123 [arXiv: 1302.5246] [INSPIRE].

[37] M. Lüscher, Future applications of the Yang-Mills gradient flow in lattice QCD, PoS (LATTICE2013) 016 (2014) [arXiv: 1308.5598] [INSPIRE].

[38] C. Monahan and K. Orginos, Locally smeared operator product expansions in scalar field theory, Phys. Rev. D 91 (2015) 074513 [arXiv:1501.05348] [InSPIRE].

[39] C. Monahan, The gradient flow in simple field theories, PoS(LATTICE2015) 052 (2016) [arXiv: 1512.00294] [INSPIRE].

[40] K. Fujikawa, The gradient flow in $\lambda \phi^{4}$ theory, JHEP 03 (2016) 021 [arXiv:1601.01578] [INSPIRE].

[41] R.A. Briceño, M.T. Hansen and C.J. Monahan, Role of the Euclidean signature in lattice calculations of quasidistributions and other nonlocal matrix elements, Phys. Rev. D 96 (2017) 014502 [arXiv: 1703.06072] [INSPIRE].

[42] R. Cicchetti and A. Faraone, Incomplete Hankel and modified Bessel functions: a class of special functions for electromagnetics, IEEE Trans. Antennas Propagat. 52 (2004) 3373.

[43] D.S. Jones, Incomplete Bessel functions. I, Proc. Edinburgh Math. Soc. 50 (2007) 173.

[44] F.E. Harris, Incomplete Bessel, generalized incomplete gamma, or leaky aquifer functions, J. Comput. Appl. Math. 215 (2008) 260.

[45] J. Karpie, K. Orginos, A. Rothkopf and S. Zafeiropoulos, Reconstructing parton distribution functions from Ioffe time data: from Bayesian methods to neural networks, JHEP 04 (2019) 057 [arXiv: 1901.05408] [INSPIRE].

[46] Y.-Q. Ma and J.-W. Qiu, Exploring partonic structure of hadrons using ab initio lattice QCD calculations, Phys. Rev. Lett. 120 (2018) 022003 [arXiv:1709.03018] [INSPIRE].

[47] Y.-Q. Ma and J.-W. Qiu, Extracting parton distribution functions from lattice QCD calculations, Phys. Rev. D 98 (2018) 074021 [arXiv:1404.6860] [INSPIRE].

[48] Z.-Y. Li, Y.-Q. Ma and J.-W. Qiu, Extraction of next-to-next-to-leading-order PDFs from lattice QCD calculations, arXiv:2006.12370 [INSPIRE].

[49] L.-B. Chen, W. Wang and R. Zhu, Next-to-next-to-leading order corrections to quark quasi parton distribution functions, arXiv:2006.14825 [INSPIRE].

[50] V.M. Braun, K.G. Chetyrkin and B.A. Kniehl, Renormalization of parton quasi-distributions beyond the leading order: spacelike vs. timelike, JHEP 07 (2020) 161 [arXiv:2004.01043] [INSPIRE]. 
[51] L.-B. Chen, W. Wang and R. Zhu, Quasi parton distribution functions at NNLO: flavor non-diagonal quark contributions, Phys. Rev. D 102 (2020) 011503 [arXiv:2005.13757] [INSPIRE].

[52] L.-B. Chen, W. Wang and R. Zhu, Master integrals for two-loop QCD corrections to quasi PDFs, arXiv:2006.10917 [INSPIRE].

[53] NNPDF collaboration, Parton distributions from high-precision collider data, Eur. Phys. J. C 77 (2017) 663 [arXiv: 1706.00428] [InSPIRE].

[54] S. Dulat et al., New parton distribution functions from a global analysis of quantum chromodynamics, Phys. Rev. D 93 (2016) 033006 [arXiv:1506.07443] [INSPIRE].

[55] S. Alekhin, J. Blümlein, S. Moch and R. Placakyte, Parton distribution functions, $\alpha_{s}$, and heavy-quark masses for LHC run II, Phys. Rev. D 96 (2017) 014011 [arXiv:1701.05838] [INSPIRE].

[56] A.D. Martin, W.J. Stirling, R.S. Thorne and G. Watt, Parton distributions for the LHC, Eur. Phys. J. C 63 (2009) 189 [arXiv:0901.0002] [INSPIRE].

[57] A. Buckley et al., LHAPDF6: parton density access in the LHC precision era, Eur. Phys. J. C 75 (2015) 132 [arXiv:1412.7420] [INSPIRE].

[58] A. Radyushkin, Nonperturbative evolution of parton quasi-distributions, Phys. Lett. B 767 (2017) 314 [arXiv: 1612.05170] [INSPIRE].

[59] X. Ji, J.-H. Zhang and Y. Zhao, More on large-momentum effective theory approach to parton physics, Nucl. Phys. B 924 (2017) 366 [arXiv:1706.07416] [INSPIRE]. 